\title{
Clasificación de la gestión del agua de uso urbano en ciudades medias de Sinaloa en México (2010-2012)*
}

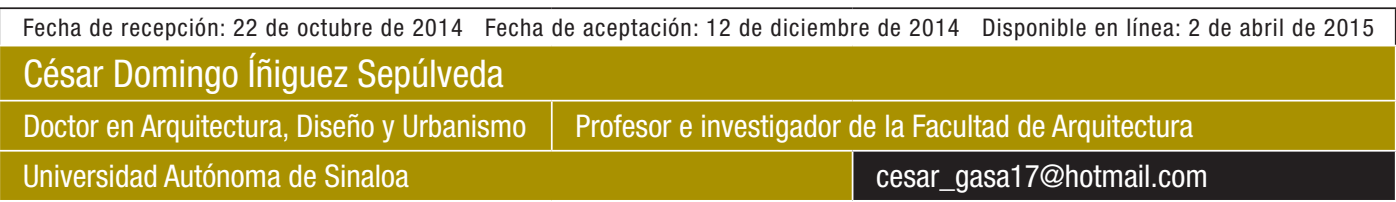

Rafael Antonio Olmedo Pérez

Doctor en Informática $\quad$ Profesor e investigador de la Facultad de Ciencias Físico Matemáticas

Universidad Autónoma de Sinaloa

rolmedo@edu.uasnet.mx

Teodoro Bernal Salazar

\begin{tabular}{|l|l|}
\hline Doctorando en Arquitectura, Diseño y Urbanismo & Profesor e investigador de la Facultad de Arquitectura
\end{tabular}

Universidad Autónoma de Sinaloa

tbernal682@hotmail.com

Yazmín Paola Î́niguez Ayón

Doctoranda en Planeación y Desarrollo Sustentable

yazpa1981@hotmail.com

Resumen Esta investigación tiene como objetivo encontrar la clasificación de los organismos operadores de los servicios de agua potable y saneamiento (OOSAPyS) de cinco ciudades con población mayor a 50.000 habitantes de Sinaloa (México). Se fundamenta en el paradigma del desarrollo sustentable (DS). Se aplicó el proceso analítico jerárquico (AHP) y se recurrió a diversas herramientas informáticas: Expert Choice (E-C) y el Decision Lab y un sistema de información geográfico (SIG) denominado Mapinfo. Los resultados obtenidos muestran una jerarquía de tres ciudades que presentan población mayor a 200.000 habitantes, en el orden de alto a medio; en cambio, se observa que ciudades con población menor a los 200.000 habitantes están en la categoría de bajo en materia de la gestión del recurso hídrico. Se conoce que el paradigma del DS orienta al manejo equitativo de agua urbana para satisfacer necesidades de generaciones actuales y futuras.

Palabras clave agua potable; gestión de agua; servicios públicos y sistema de indicadores

\footnotetext{
Este artículo se deriva del proyecto de investigación denominado "Estudio comparativo de valoración de la gestión de los servicios públicos del agua en ciudades de Sinaloa", financiado por la Universidad Autónoma de Sinaloa por medio del Programa de Fomento y Apoyo a Proyectos de Investigación (Profapi), con registro Profapi 2012/080. Fue ejecutado entre 2012 y 2013. Los investigadores participantes: César Domingo Iñiguez Sepúlveda, responsable del proyecto; Rafael A. Olmedo Pérez, Teodoro Bernal Salazar, Yazmín P. Î̃iguez Ayón, Noemí del Carmen Ramos Escobar, Rafael Sáenz Castañeda. Expresamos nuestro reconocimiento para el alumno Otoniel Olivares Soto, estudiante de la Facultad de Arquitectura y prestador de servicio social, por su compromiso con esta investigación, al realizar importantes actividades de recopilación y procesamiento de la información.
}

Cómo citar este artículo: Î́iguez-Sepúlveda, C. D., Olmedo-Pérez, R. A., Bernal-Salazar, T., \& Îñiguez Ayón, Y. P. (2015). Clasificación de la gestión del agua de uso urbano en ciudades medias de Sinaloa en México (2010-2012). Cuadernos de Vivienda y Urbanismo, 8(15), 20-35. http://dx.doi.org/10.11144/Javeriana.cvu8-15.cgau 


\section{Classification of Urban Water Management in Mid-Sized Sinaloa Cities in Mexico (2010-2012)}

Abstract This research aims to find the classification of the potable water and sanitation service providers (OOSAPyS) of five cities located in Sinaloa (Mexico) with populations greater than 50,000 inhabitants. It is based on the paradigm of sustainable development (SD). The analytic hierarchy process (AHP) was applied and various software tools were used: Expert Choice (E-C), Decision Lab and a geographic information system (GIS) called Mapinfo. The results obtained show a hierarchy of three cities with populations over 200,000 inhabitants in a high to medium order, whereas it is observed that cities with less than 200,000 inhabitants are in the category low in terms of water supply management. It is known that the paradigm of SD leads to an equitable management of urban water in order to meet the needs of present and future generations.

Keywords potable water; water management; utilities and system indicators

\section{Classificação da gestão da água de uso urbano em cidades médias de Sinaloa no México (2010-2012)}

Resumo Esta pesquisa tem como objetivo encontrar a classificação dos organismos operadores dos serviços de água potável e saneamento (OOSAPeS) de cinco cidades com população maior de 50.000 habitantes de Sinaloa (México). Baseia-se no paradigma do desenvolvimento sustentável (DS). O processo analítico hierárquico (AHP) foi aplicado e recorreuse a diversas ferramentas informáticas: Expert Choice (E-C), o Decision Lab e um sistema de informação geográfica (SIG) nomeado Mapinfo. Os resultados obtidos mostram uma hierarquia de três cidades que apresentam população maior de 200.000 habitantes, em ordem de mais alto para meio; no entanto observa-se que cidades com população menor aos 200.000 habitantes estão na categoria de baixo na questão da gestão do recurso hídrico. Sabe-se que o paradigma DS orienta o maneio equitativo da água urbana para satisfazer necessidades de geraçóes presentes e futuras. 


\section{Introducción}

El Banco Mundial patrocinó en la década de 1990 la creación de la Red Internacional para la Comparación de Entidades de Agua Potable y Saneamiento (Rieapos), cuyo propósito es ofrecer información comparativa entre las empresas dedicadas a operar o regular el abastecimiento a sistemas urbanos. Rieapos ofrece a los organismos asociados un software para capturar y evaluar de manera homogénea un conjunto mínimo de indicadores de gestión (Van den Berg y Danilenko, 2010).

En el Plan Nacional de Desarrollo 2007-2012, el Gobierno de México asumió como política central el desarrollo humano sustentable, lo que significa pugnar porque todos los mexicanos alcancemos altos índices de bienestar sin comprometer las capacidades de las siguientes generaciones. Esto quedó expresado en el Programa Nacional Hídrico 2007-2012 (PNH 2007-2012), que establece muy ambiciosos objetivos y metas en rubros como sustitución de fuentes de suministro, incremento de cobertura de los servicios de agua potable y alcantarillado, tratamiento de las aguas residuales y mejoramiento de la eficiencia del uso del agua en el sector agrícola (Secretaría de Medio Ambiente y Recursos Naturales, 2011).

Los servicios de agua potable y saneamiento son responsabilidad de un Estado garante, ya sea que provea los servicios de manera directa, que sea un prestador más o que solo regule el ámbito económico del servicio provisto por particulares. En los últimos cien años, la satisfacción de esos servicios ha sido un asunto personal, una actividad de las comunidades locales, un servicio administrado por el Gobierno federal descentralizado a los ayuntamientos de los municipios. Asimismo, puede ser prestado por particulares, en teoría regulados por ese Estado garante, aunque no siempre bajo condiciones claras (Collado, 2008).

La caracterización de un sistema ecológico-ambiental, social, económico e institucional que es objeto de gestión constituye un paso clave en el proceso de evaluación de la sostenibilidad de tal sistema. Dicha caracterización implica subdividir el sistema en sus diferentes componentes, con el fin de trazar la red de relaciones que subyace al funcionamiento del mismo, así como las relaciones causa-efecto o interacciones responsables de potenciales deficiencias o sinergias en el sistema (Settle, Crocker y Shogren, 2002).

El diseño y la implementación de un marco de análisis de este tipo requiere la aplicación de técnicas y metodologías de muy diversa índole (técnicas participativas, técnicas de análisis multicriterio (AMC), análisis de costo-beneficio, gestión de datos, aplicación en la construcción de modelos cualitativos y cuantitativos y en otros campos, por lo que desde el punto de vista científico-técnico también debe concebirse a partir de la perspectiva multidisciplinar e integradora (Urzelai et al., 2006).

Conforme a lo dicho, se expondrá y aplicará una de las técnicas multicriterio más extendidas: el proceso analítico jerárquico (AHP, por su nombre en inglés: Analytic Hierarchy Process), propuesto por Saaty en 1980. Aunque esta técnica empezó a explorarse de manera exhaustiva desde 
la década del ochenta, en busca de alternativas en el campo de estudios de investigación cuantitativos y cualitativos, todavía se recurre a ella porque brinda solución a problemas de carácter multicriterio, en los que se evalúa una serie de opciones respecto a un conjunto de criterios.

\section{Fundamento metodológico}

En el pasado, la toma de decisiones para escoger las mejores alternativas de administración de organismos operadores de agua de ciudades se basaba solo en criterios económicos, sobre todo en la minimización de los costos. Hoy, uno de los retos de tomadores de decisión en los países empobrecidos es construir herramientas que consideren otros aspectos importantes para el desarrollo regional y local, como las ambientales, sociales, institucionales y económicas.

La mayoría de los instrumentos que existen evalúa criterios técnicos y económicos. Estos mecanismos suelen basarse en programas informáticos que parten del diseño o de la generación de diversas opciones para la gestión del agua que se distribuye entre la población de los entornos urbanos y rurales. Luego examinan las alternativas, las ponderan y las comparan.

En la literatura de la toma de decisión multicriterio se abordan tres tipos de situaciones problemáticas:

a. Selección de la mejor alternativa.

b. Clasificación de las alternativas existentes (de la mejor a la peor).

c. Clasificación de las alternativas en grupos homogéneos.
Durante las últimas tres décadas se han propuesto diferentes técnicas de clasificación multicriterio, entre las que se halla el proceso analítico jerárquico. Como la mayoría de las grandes ideas científicas, el AHP puede considerarse de muy diversas maneras, según la orientación dada al mismo. Su contribución es importante en ámbitos operativos, tácticos y estratégicos y sirve para mejorar la eficiencia, la eficacia y, sobre todo, la efectividad del sistema. En resumen, se puede entender el AHP como:

a. Una técnica que permite la resolución de problemas multicriterio, multientorno y multiactores. Incorpora los aspectos tangibles e intangibles en el modelo, así como el subjetivismo y la incertidumbre inherente en el proceso de toma de decisiones.

b. Una teoría matemática de la medida generalmente aplicada a la dominación de la influencia entre alternativas respecto a un criterio o atributo ${ }^{1}$.

c. Una filosofía para abordar la toma de decisiones.

Lo anterior muestra las ideas intuitivas en las que se basa la filosofía del AHP y los fundamentos teóricos que soportan esta teoría matemática, reconocida como una técnica de decisión multicriterio. A continuación se describen las tres etapas del proceso metodológico del AHP: modelación, valoración y priorización y síntesis (Saaty, 1980), propuestas desde su formulación inicial.

a. En la primera etapa, modelización, se construye un modelo o una estructura en la que se representen todos los aspectos relevantes en el proceso de resolución (actores, escenarios, factores, elementos e interdependencias). 
b. En la segunda etapa, valoración, se incorporan preferencias, gustos y deseos de los actores mediante los juicios incluidos en las matrices de comparaciones pareadas. Estas matrices cuadradas $\mathrm{A}=$ (aij) reflejan la dominación relativa que representa la dominación ${ }^{2}$ de la alternativa $i$ sobre la $j$. En su construcción se plasma el pensamiento y el proceder del profesor Saaty al medir aspectos intangibles.

c. La última etapa de la metodología, priorización y síntesis, proporciona las prioridades consideradas en la resolución del problema: prioridades locales; prioridades globales y prioridades totales. En general, se entiende por prioridad una unidad abstracta válida para cualquier escala en la que se integran las preferencias que el individuo tiene al comparar aspectos tangibles e intangibles.

En este caso, la matriz recíproca de comparaciones pareadas $\mathrm{W}=(\mathrm{wi} / \mathrm{wj})$ queda como se muestra en la Figura 1:

\section{Figura 1.}

Matriz de (W) para comparaciones pareadas

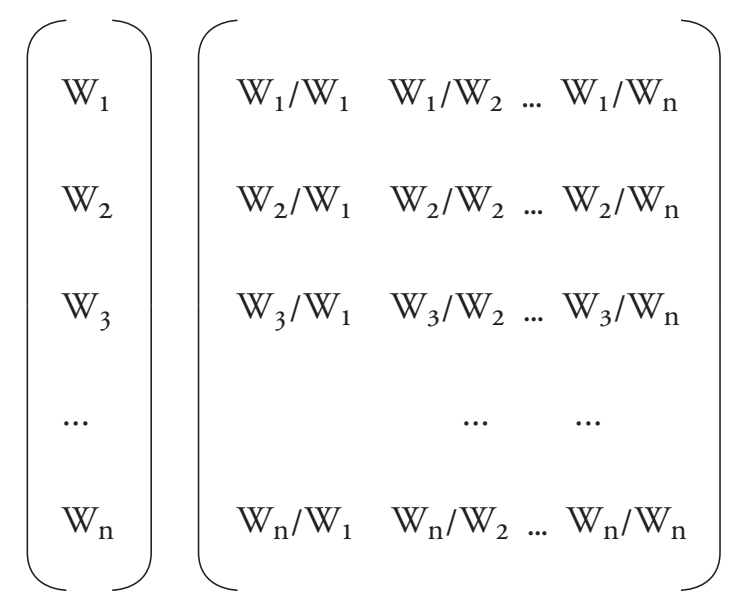

Fuente: elaboración propia
La matriz W tiene rango 1, con lo que el problema del autovector se reduce a $\mathrm{WW}_{\mathrm{W}}=n \mathrm{w}$, con $\sum \mathrm{j} w \mathrm{j}=1$. Una forma sencilla de obtener el valor de $\lambda \max$ si se conoce el valor exacto de $w$ (o estimación) en forma normalizada es sumar las columnas de A y multiplicar el vector resultante por el vector de prioridades $w$. Al utilizar el teorema de Perron-Frobenius, se puede probar que $\lambda \max \geq n$ para el método de Saaty (1980).

Una de las grandes ventajas del AHP es que relaja las hipótesis tan restrictivas que imponía el enfoque tradicional en la decisión (escuela utilitarista); en concreto, no exige la transitividad en las preferencias. Además, evalúa el grado de consistencia del decisor a la hora de introducir los juicios en las matrices recíprocas de comparaciones pareadas.

En AHP se dice que el decisor o la persona que introduce los juicios es consistente si la matriz de comparaciones pareadas lo es, esto es, si verifica que $(a i j)(a j k)=a i k, \forall i, j, k$. Para evaluar la consistencia del decisor, se calcula la denominada razón de consistencia (RC), un índice no estadístico (en su propuesta inicial) que viene dado como el cociente entre el Índice de Consistencia (IC) y el Índice de Consistencia Aleatorio (ICA), multiplicado por $n$, esto es:

$$
\mathrm{RC}=\frac{\mathrm{IC}}{\mathrm{ICA}(n)}
$$

Donde $\mathrm{a} i j=a i j w j / w i$, el ICA medio obtenido al simular aleatoriamente los juicios para las matrices recíprocas de orden $n$. Los valores del Índice de Consistencia Aleatorio para la diferente $n$, obtenidos mediante la simulación de $100.000 \mathrm{ma}$ trices (Aguarón y Moreno-Jiménez, 2003), son como se describe en la Tabla 1. 
Tabla 1.

Valores del Índice de Consistencia Aleatoria (ICA)

\begin{tabular}{ccccccccccc}
$N$ & 3 & 4 & 5 & 6 & 7 & 8 & 9 & 10 & 11 & 12 \\
\hline ICA & 0,525 & 0,882 & 1,115 & 1,252 & 1,341 & 1,404 & 1,452 & 1,484 & 1,513 & 1,535 \\
\hline
\end{tabular}

Fuente: Aguarón y Moreno-Jiménez (2003)

Para $n=3$, Juan Aguarón obtuvo, mediante la enumeración de todos los juicios posibles, el valor exacto de ICA (3) $=0,5245$. En la práctica, suelen darse por buenas razones de consistencia inferiores a un $10 \%{ }^{3}$. Si la razón de consistencia supera ese umbral, se recomienda revisar los juicios y corregir aquel que más se separa de la razón dada por las prioridades relativas correspondientes (comparar aij con wi/ wj).

El programa informático Expert Choice (E-CH) no solo se basa en el AHP, sino que es la concreción informática desarrollada o supervisada por Saaty de este método de análisis de decisiones multicriterio. La referencia más completa y a la vez técnica y divulgativa del AHP es de Saaty (1996), aunque hay excelentes síntesis introductorias de Zahedi (1986) y Harker (1987). Por último, se argumenta que Expert Choice es un potente, versátil, analíticamente sólido, bien documentado y amigable paquete de decisión multicriterio. Sus limitaciones se deben a lo que es su razón de ser: seguir la metodología AHP (Barba-Romero y Pmerol, 1997).

En síntesis, en esta investigación se considera que para el análisis de las alternativas se debe utilizar el método multicriterio, con el fin de obtener un equilibrio entre los impactos sociales, ambientales y técnicos al momento de escoger la mejor alternativa de solución. Aunque este estudio no es aplicado en proyectos de electrificación, la metodología utilizada es la que mejor se ajusta a las necesidades de la valoración de los distintos OOSAPyS con criterios que establece el nuevo paradigma del desarrollo sustentable y emplea, además, una escala de valoración válida tanto para aspectos cuantitativos como cualitativos.

Una reflexión importante que aquí se evidencia es el asunto del período de estudio (2010-2012). Se seleccionó este tiempo fundamentado en dos cuestiones: una, que la duración administrativa municipal en nuestro país es de solo tres años, de acuerdo con la Constitución Política de los Estados Unidos Mexicanos; la otra es que la información solo está sistematizada para ese mismo lapso en cada una de las cinco ciudades en estudio.

\section{Sistema de indicadores}

Un indicador sintético puede definirse como "aquella función matemática de los indicadores parciales que reúne un conjunto de condiciones que se juzgan necesarias para concretar una medición expresiva del objetivo buscado" (Pena, 1978, p. 66) o, lo que es lo mismo, una función de un conjunto de variables e indicadores intermedios, cada una de las cuales contribuye a cuantificar algún aspecto del concepto para el que se quiere cuantificar su magnitud (López, Sánchez e Iglesias, 2003).

Los indicadores sintéticos son creados a partir de los modelos formales del sistema en representación de la realidad en estudio. Sin embargo, el proceso de formulación de indicadores sintéticos no solo expresa las características del fenómeno; también incluye decisiones tomadas por el 
analista en relación con la observación de la fenómeno (Pérez et al., 2009).

Se considera aquí la propuesta de construcción de un sistema inicial de indicadores $m$ para evaluar un conjunto de $n$ unidades. Hay que distinguir entre indicadores positivos, negativos y neutrales; si la dirección mejora, empeora o permenece, así sucederá con su posición final en la clasificación del sistema de indicadores, de manera que "positivo es mejor" o "negativo es peor". El indicador se consideró positivo cuando un mayor valor representó una mejora en la gestión de servicios del agua de uso urbano, al aplicar principios y criterios del desarrollo sustentable. Por lo contrario, el indicador es negativo cuando su valor representa un quebranto en la administración de los OOSAPyS desde la propia sostenibilidad del recurso hídrico y se considera neutral cuando no tiene una dirección específica de mejora o deterioro en el proceso de la sustentabilidad.
En primer término, se tomó en cuenta que los valores más altos representan una mayor sustentabilidad en la gestión de los OOSAPyS y que los pesos introducidos en el indicador son en dirección positiva. En segundo lugar, el sistema de ponderación nos permite identificar los aspectos que tienen mayor influencia en la sostenibilidad y que, por lo tanto, deben considerarse al planificar las políticas, estrategias y acciones de los organismos operadores del agua.

Se debe señalar que, a pesar de sus ventajas, el uso de la distancia en la definición del indicador compuesto hace que los resultados finales sean determinados por el conjunto de unidades que se examinan y así el analista debe llevar a cabo una selección apropiada de los indicadores. En la Tabla 2 se detalla la estructura del sistema global de indicadores para valorar los OOSAPyS en las ciudades de Mazatlán, Culiacán, Guamúchil, Guasave y Los Mochis.

Tabla 2.

Sistema global de indicadores

\begin{tabular}{|c|c|c|c|c|}
\hline Indicador & Definición & Dimensión & Signo & Evaluación \\
\hline $\mathrm{IS}_{1}$ & Población total en el espacio territorial & Social & Negativo & Directa \\
\hline $\mathrm{IS}_{2}$ & Población atendida con servicio de Agua Potable (AP) & Social & Positivo & Directa \\
\hline $\mathrm{IS}_{3}$ & Población urbana atendida con servicio de alcantarillado & Social & Positivo & Directa \\
\hline $\mathrm{IS}_{4}$ & Población urbana atendida con servicio de saneamiento & Social & Positivo & Directa \\
\hline $\mathrm{IS}_{5}$ & Cobertura de la población con servicio de AP & Social & Positivo & Directa \\
\hline $\mathrm{IS}_{6}$ & Cobertura de la población atendida con servicio de alcantarillado & Social & Positivo & Directa \\
\hline $\mathrm{IS}_{7}$ & Cobertura de la población atendida con servicio de saneamiento & Social & Positivo & Directa \\
\hline $\mathrm{IS}_{8}$ & Dotación de agua potable & Social & Positivo & Directa \\
\hline $\mathrm{IMA}_{1}$ & $\begin{array}{l}\text { Caudal producido mediante la extracción del agua } \\
\text { de fuentes superficiales y subterráneas }\end{array}$ & Ambiental & Positivo & Directa \\
\hline $\mathrm{IMA}_{2}$ & Caudal desinfectado & Ambiental & Positivo & Directa \\
\hline $\mathrm{IMA}_{3}$ & Caudal potabilizado & Ambiental & Positivo & Directa \\
\hline $\mathrm{IMA}_{4}$ & Capacidad instalada AP & Ambiental & Positivo & Directa \\
\hline $\mathrm{IMA}_{5}$ & Agua no contabilizada (fugas del sistema) & Ambiental & Positivo & Directa \\
\hline
\end{tabular}




\begin{tabular}{ccccc}
\hline $\mathrm{IMA}_{6}$ & Caudal producido Aguas Residuales Municipales (ARM) & Ambiental & Positivo & Directa \\
\hline $\mathrm{IMA} \mathrm{A}_{7}$ & Capacidad instalada ARM & Ambiental & Positivo & Directa \\
\hline $\mathrm{IMA} \mathrm{A}_{8}$ & Caudal tratado ARM & Ambiental & Positivo & Directa \\
\hline $\mathrm{E}_{1}$ & Cobertura de macromedición en fuente de producción & Económica & Positivo & Directa \\
\hline $\mathrm{IE}$ & Cobertura de micromedición doméstica & Económica & Positivo & Directa \\
\hline $\mathrm{IE}_{3}$ & Cobertura de micromedición comercial & Económica & Positivo & Directa \\
\hline $\mathrm{IE}_{4}$ & Cobertura de micromedición industrial & Económica & Positivo & Directa \\
\hline $\mathrm{IE}_{5}$ & Eficiencia física & Económica & Positivo & Directa \\
\hline $\mathrm{IE}_{6}$ & Eficiencia comercial & Económica & Positivo & Directa \\
\hline $\mathrm{IE}_{7}$ & Eficiencia global & Económica & Positivo & Directa \\
\hline $\mathrm{IE}_{8}$ & Tarifa doméstica con rango de consumo de 10 a $20 \mathrm{~m}^{3}$ & Económica & Negativo & Directa \\
\hline
\end{tabular}

Fuente: elaboración propia

\section{Resultados y análisis de 00SAPyS ${ }^{4}$ de ciudades de Sinaloa}

Con el propósito de cumplir con el objetivo de la Comisión Nacional del Agua (Conagua) de "incrementar el acceso y calidad de los servicios de agua potable, alcantarillado y saneamiento", es de suma importancia contar con un sistema de indicadores que reflejen la situación imperante en las entidades prestadoras de dichos servicios y con ellos evaluar la gestión de los OOSAPyS en ciudades distribuidas en la geografía de Sinaloa.

Esta información permitirá a los prestadores de servicios conocer la forma como operan y los grados de eficiencia bajo los cuales están actuando para compararlos con otros organismos. La posibilidad de comparar sus resultados con los de otro prestador de servicios de Sinaloa y de México será de gran utilidad, pues incentivará y facilitará la toma de medidas necesarias a fin de mejorar su desempeño y brindar un mejor servicio entre la población usuaria de los servicios que ofrece.

Al Gobierno estatal, la información le ofrecerá una visión general del desempeño de las entidades prestadoras de los Sapas de las cinco ciudades más importantes del estado de Sinaloa y le simplificará la toma de decisiones en la orientación de su política sectorial, para mejorar su apoyo a los prestadores de servicios, con el fin de que estos brinden un servicio más eficiente y de buena calidad encaminado a elevar la calidad de vida de la población.

En la instrumentación de esta metodología de valoración basada en indicadores fue fundamental la veracidad de su información. La información que se utilizó en la construcción de los indicadores de desempeńo fue recopilada a partir de los datos publicados por la Conagua, en su publicación anual denominada Situación del subsector agua potable, alcantarillado y saneamiento, tomada entre los ańos 2010 y 2012, así como los datos generados por los propios organismos

4 Con estas siglas (OOSAPyS) denotamos a los Organismos Operadores Prestadores de los Servicios de Agua Potable y Saneamiento. Son dependencias de gobiernos municipales, estatales o federales que, de acuerdo con la Ley de Aguas Nacionales de 2004 vigente, están facultados para prestar los servicios públicos de agua potable, alcantarillado y saneamiento, bien sea de manera directa o mediante concesión a empresas privadas. 
operadores municipales de las cinco ciudades en estudio (Conagua, 2010; 2011; 2012).

La obtenida corresponde a las cinco ciudades prestadoras de los servicios de agua potable y saneamiento en las localidades que funcionan como Alcaldías centrales de cada municipio que albergan, en promedio, un $75 \%$ de su población municipal. En el proceso de recolección, algunos de los organismos operadores reportaron información y, en otros casos, existen datos inconsistentes.

A continuación se presentan las figuras que exponen la dinámica de la gestión de los OOSAPyS en las cinco principales ciudades del estado de Sinaloa desde 2010 hasta 2012, en atención a la recopilación para los veinticuatro indicadores de las tres categorías y también se le ofrecen

Figura 2.

INDICE GESTIÓN DE SAPAS 2010. Valoración de la categoría social para el año 2010

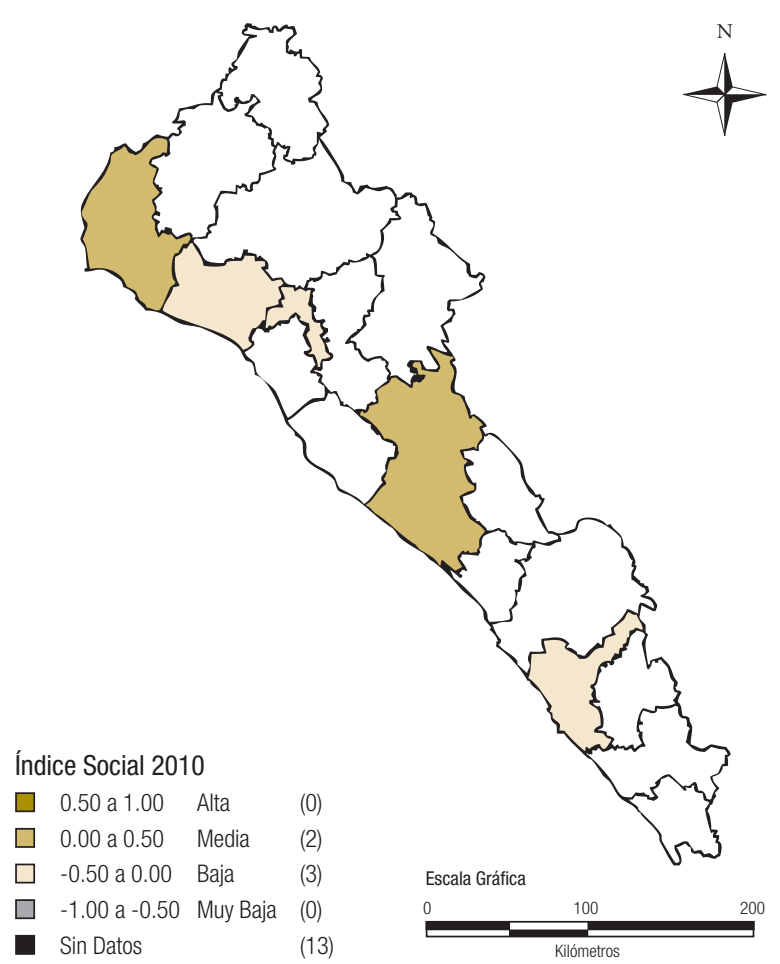

Fuente: elaboración propia imágenes que muestran el resultado de los tres soportes del desarrollo sustentable.

La Figura 2 señala el estado del índice de valoración de la categoría social para 2010. Vemos que las ciudades de Culiacán y Los Mochis presentan un nivel medio del índice de valoración; en cambio, Guasave, Guamúchil y Mazatlán están ubicados en un nivel bajo en el índice de gestión del recurso hídrico entre los OOSAPyS. Aquí es fundamental que los OOSAPyS de Guasave, Guamúchil y Mazatlán instrumenten estrategias para mejorar el desempeño de los indicadores que componen la categoría social y alcancen el siguiente nivel en la clasificación de valoración de gestión del agua de uso en el contexto urbano.

La Figura 3 nos permite deducir que entre 2010 y 2011 no hubo cambios reveladores del desempeño

Figura 3.

INDICE GESTIÓN DE SAPAS 2010. Valoración de la categoría social para el año 2011

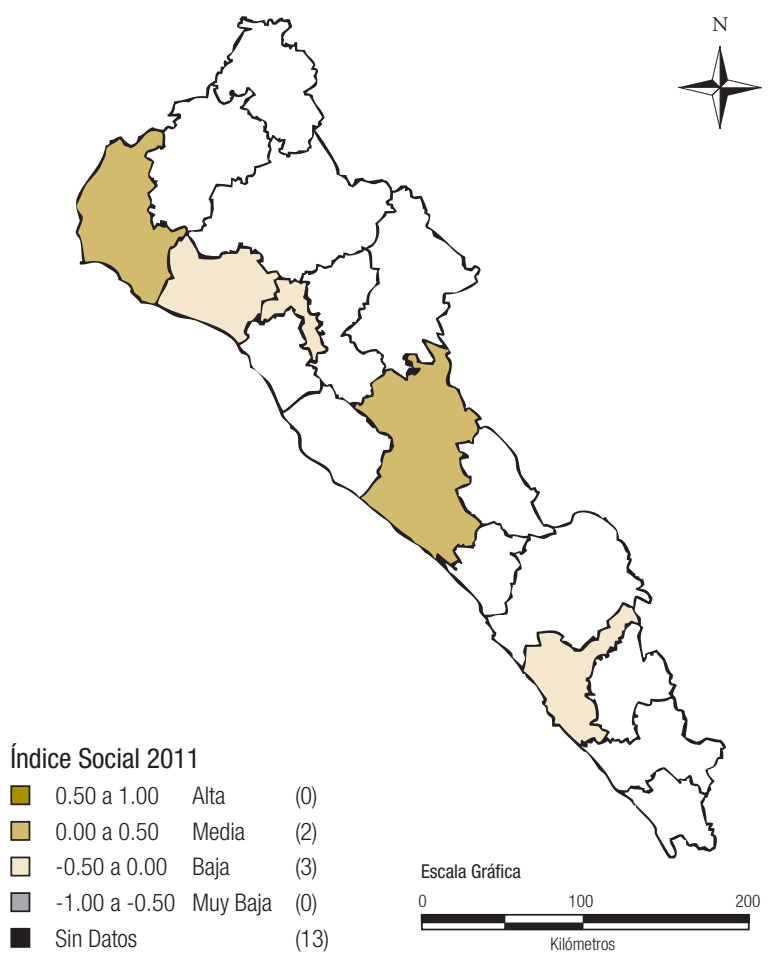

Fuente: elaboración propia 
de los OOSAPyS en la categoría social, es decir, se mantienen las ciudades de Culiacán y Los Mochis en el nivel medio. De igual manera, se observa que las ciudades de Guasave, Guamúchil y Mazatlán conservan nivel bajo, en materia de la gestión realizada por los OOSAPyS con el agua de uso urbano para 2011. De la misma manera, se hace la observación para que los OOSAPyS de las ciudades Guasave, Guamúchil y Mazatlán empleen mecanismos entre los indicadores que componen la categoría social para que optimen el desempeño y obtengan el siguiente nivel en la clasificación de valoración de gestión del agua de uso en el contexto urbano.

En la Figura 4 se observa el momento para el año 2012. Aquí hay solo un cambio significativo en sentido positivo, al pasar de un nivel inferior a uno superior de la clasificación y esta es la ciudad

Figura 4

INDICE GESTIÓN DE SAPAS 2010. Valoración de la categoría social para el año 2012

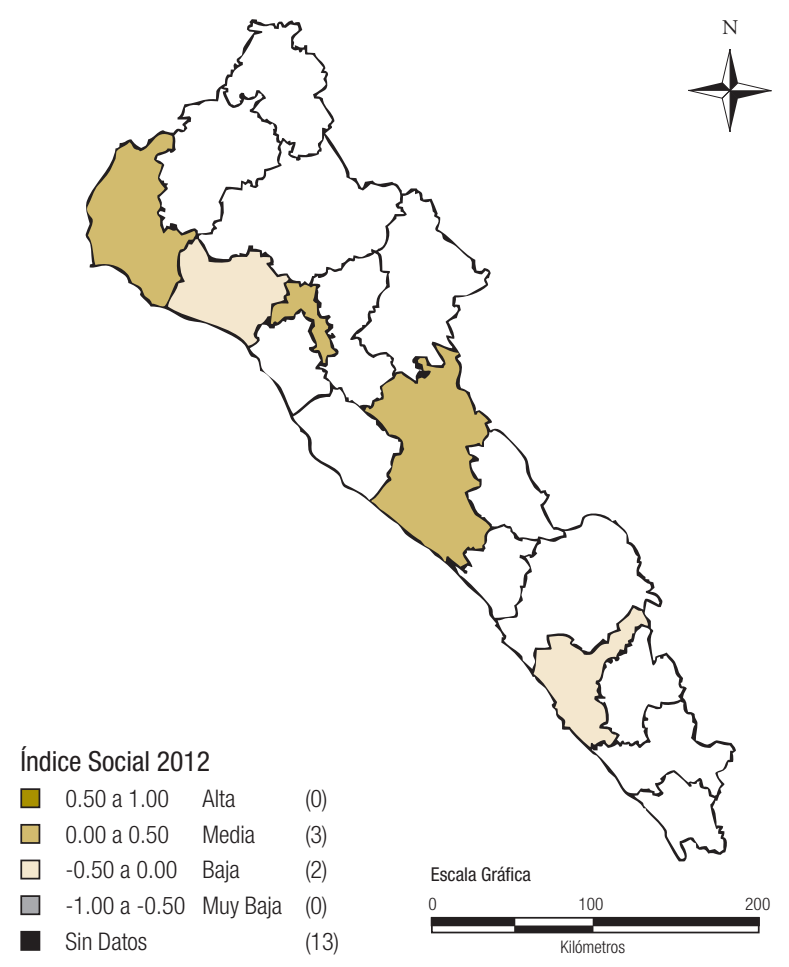

de Guamúchil y se agrega a las ciudades de $\mathrm{Cu}$ liacán y Los Mochis en el nivel medio de la valoración del índice de gestión de los OOSAPyS; en cambio, las ciudades de Guasave y Mazatlán mantienen su nivel bajo.

Por el contrario, en la Figura 5 se describe el ordenamiento de la gestión del recurso hídrico acaecido en 2010 para el indicador ambiental; solo Culiacán está en la posición de alta gestión del agua de uso urbano. En contraste, Los Mochis y Mazatlán se ubican en la categoría de media en atención a la gestión del agua, mientras Guasave y Guamúchil muestran una gestión del recurso hídrico de bajo nivel. Desde luego, es necesario expresar una observación importante: los OOSAPyS deben fomentar estrategias de administración en los indicadores de la categoría ambiental, a fin de que se mejore el

\section{Figura 5.}

INDICE GESTIÓN DE SAPAS 2010. Valoración de la categoría ambiental, 2010

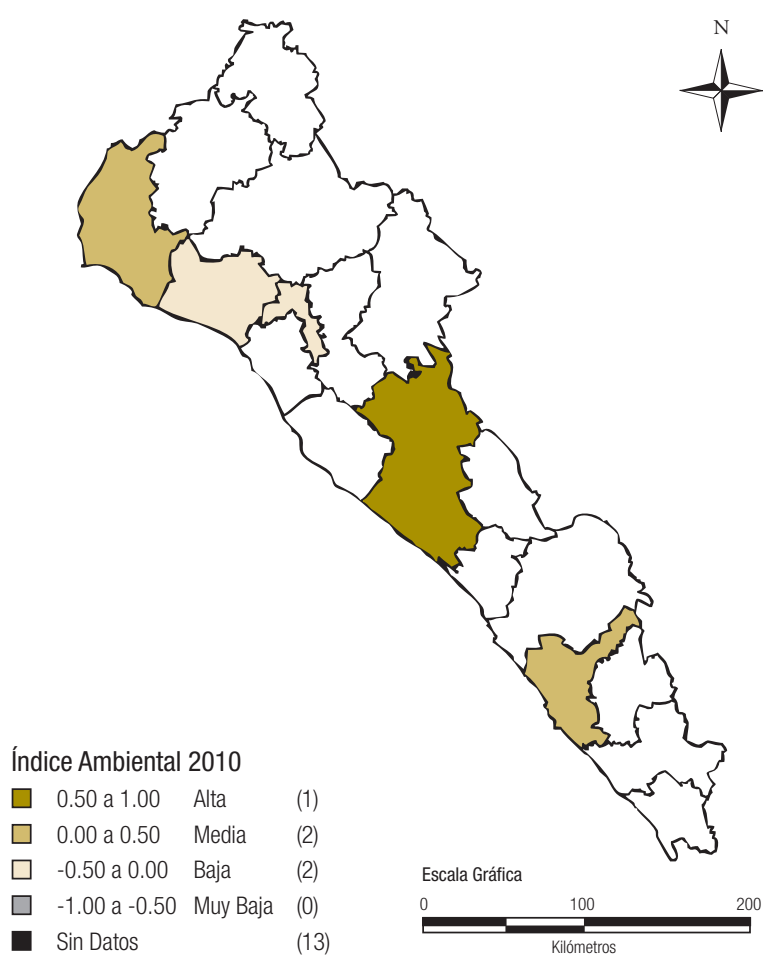


desempeño de la gestión del recurso agua y estas ciudades puedan acceder a una calificación superior en la clasificación.

La gestión de líquido vital de uso urbano para 2011 se aprecia en la Figura 6. Aquí se observa que dos ciudades y sus regiones, Culiacán y Mazatlán, están catalogadas en el nivel alto. En contraste, Los Mochis, Guasave y Guamúchil se encuentran en la categoría de baja gestión realizada por los OOAPYS en materia del recurso hídrico, lo que orienta a que se implementen estrategias que mejoren el desempeño de los indicadores de la categoría ambiental para que estos organismos avancen al siguiente peldaño de la valoración.

El índice de la gestión de los servicios públicos del agua para el año 2012 desde la perspectiva

\section{Figura 6.}

INDICE GESTIÓN DE SAPAS 2010. Valoración de la categoría ambiental, 2011

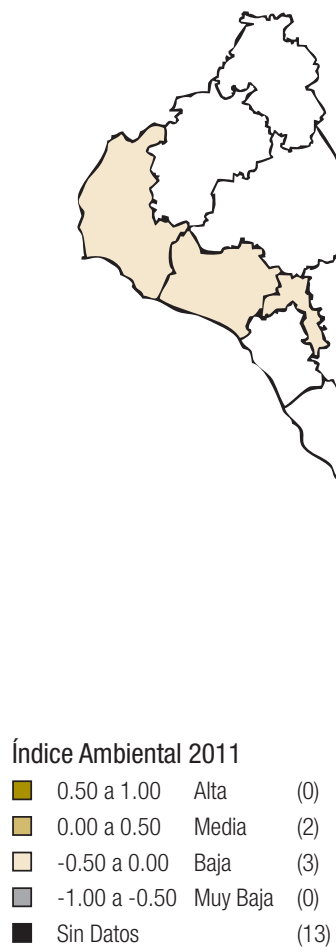

Fuente: elaboración propia de la categoría ambiental se muestra en la Figura 7. Está en la mira una configuración muy diferente al año 2011. Solo Culiacán y su municipio alcanzaron alto nivel en el índice gestión del líquido vital en los OOSAPyS. De igual manera, se observa que las ciudades de Los Mochis y Mazatlán están consideradas en nivel del índice de gestión medio y Guamúchil se ubica en un nivel muy bajo. Por lo anterior, se deben instrumentar acciones entre los indicadores de esta categoría ambiental para que mejoren su desempeño.

La Figura 8 nos indica el comportamiento del índice de gestión de los OOSAPyS en las ciudades del estado de Sinaloa para el ańo 2010 en la categoría económica. En la gráfica territorial se observa que la ciudad capital del estado, Culiacán, se encuentra en un alto nivel. De igual manera,

Figura 7.

INDICE GESTIÓN DE SAPAS 2010. Valoración de la categoría ambiental, 2012

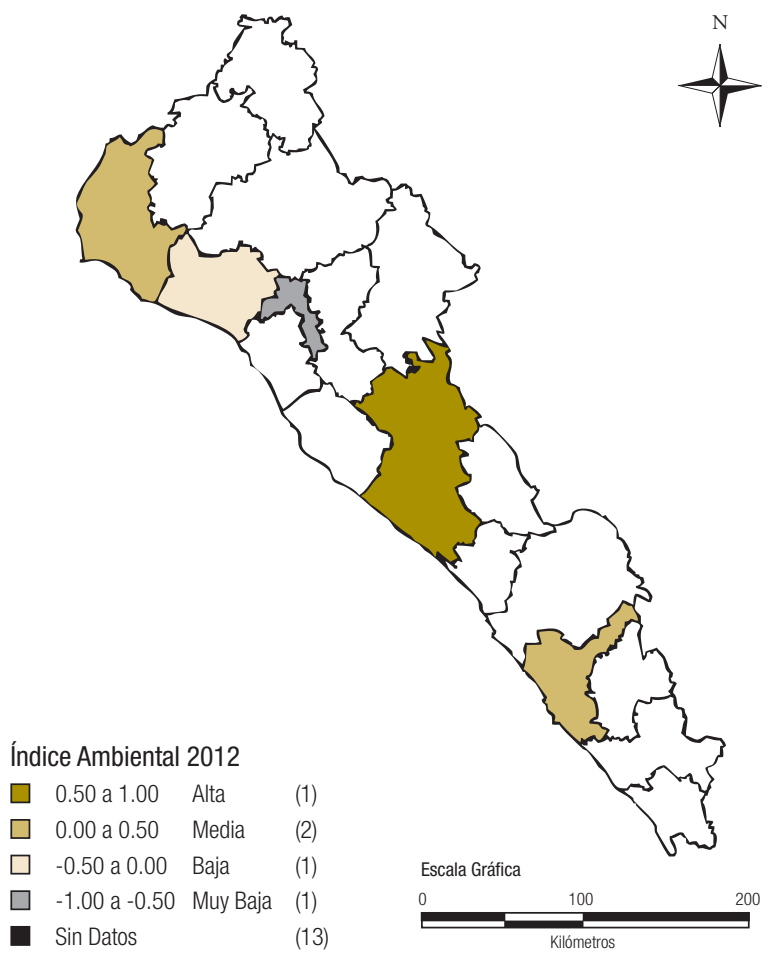

Fuente: elaboración propia 
Figura 8

INDICE GESTIÓN DE SAPAS 2010. Valoración de la categoría económica, 2010

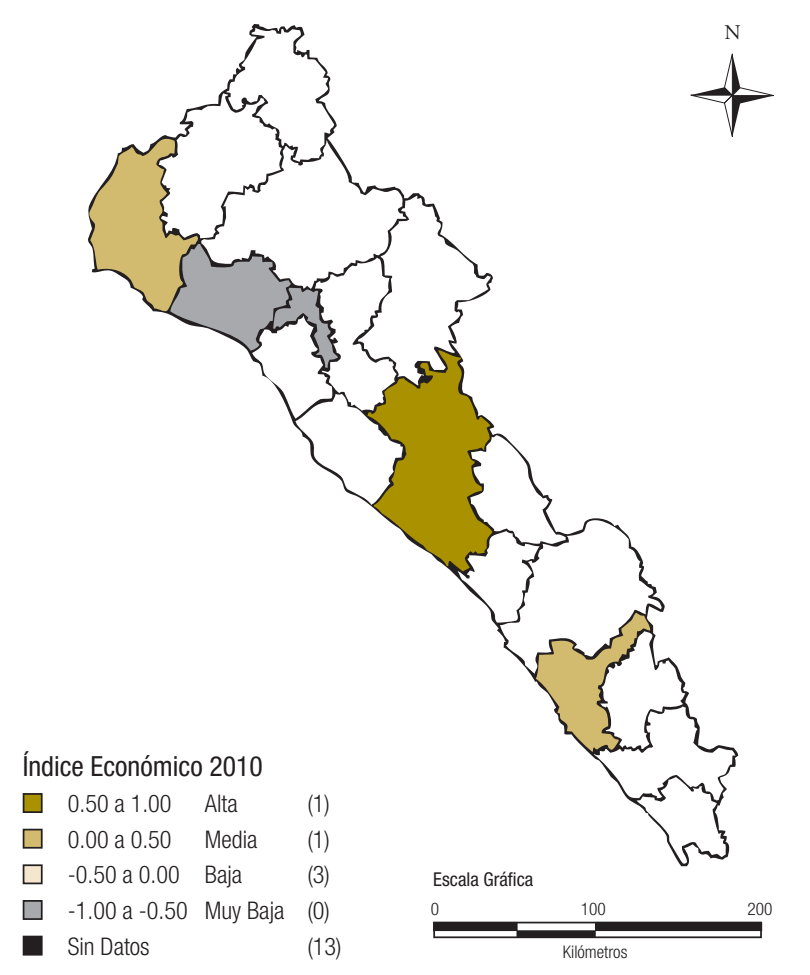

Fuente: elaboración propia

identificamos que las ciudades de Los Mochis y Mazatlán se clasifican con un valor medio en el índice de gestión del recurso hídrico. En la parte inferior, en el nivel muy bajo, están las ciudades de Guasave y Guamúchil, cuyos OOSAPyS deben mejorar su desempeño en los indicadores de la categoría económica.

La serie territorial sobre el encargo de los servicios públicos del agua para el año 2011 desde la perspectiva de la categoría económica se muestra en la Figura 9. Aquí está descrito que la ciudad capital, Culiacán, mantiene la hegemonía sobre las otras ciudades del estudio, al ubicarse en el puesto alto de la valoración. Al igual que Culiacán, Mazatlán también conserva su nivel del año anterior (2010). En contraste, Los Mochis desciende su nivel de valoración y se equipara con
Figura 9

INDICE GESTIÓN DE SAPAS 2010. Valoración de la categoría económica, 2011

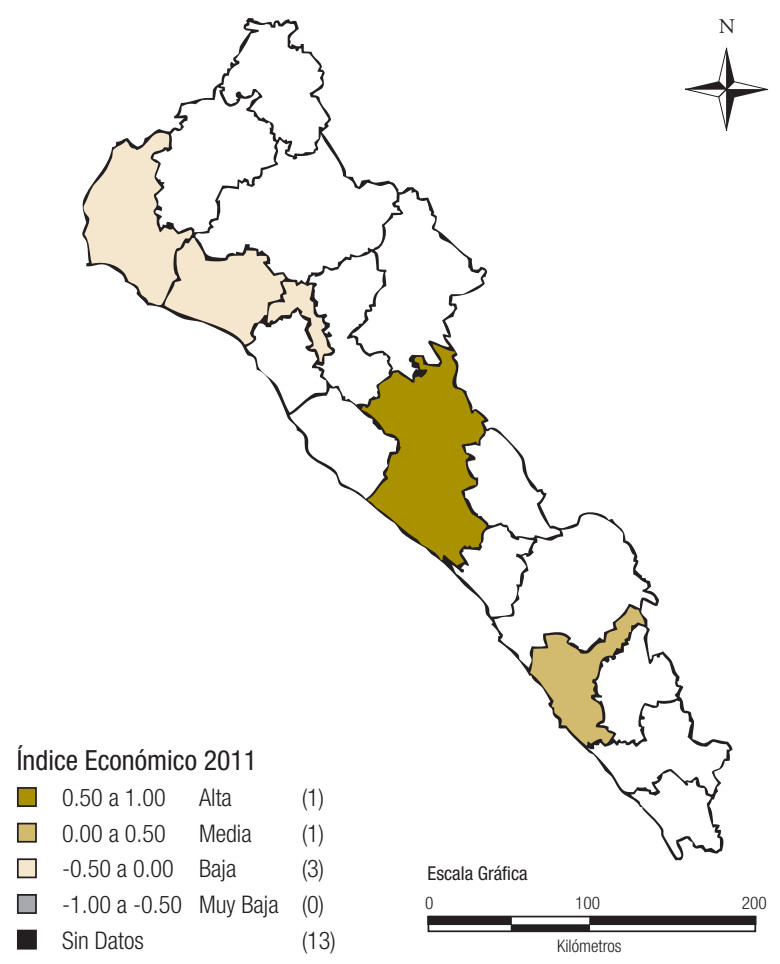

Fuente: elaboración propia

Guasave y Guamúchil, que alcanzaron un nivel de baja gestión de los OOSAPyS. En consecuencia, estas últimas localidades y sus OOSAPyS deben promover la mejora en la calidad de los indicadores de la categoría económica.

La valoración de la gestión de líquido vital de uso urbano para el año 2012 se muestra en la Figura 10. En esta imagen se observa que la ciudad de Culiacán mantiene su valoración de la gestión del recurso hídrico al ubicarse en el nivel de alta gestión. Las demás ciudades están en el nivel de baja gestión del agua; se mantuvieron en esta categoría las ciudades de Los Mochis, Guasave y Guamúchil, mientras Mazatlán disminuyó un peldaño. En estas últimas ciudades, los OOSAPyS deben impulsar mejoras de los indicadores de la categoría económica. 
Figura 10.

INDICE GESTIÓN DE SAPAS 2010. Valoración de la categoría

económica, 2012

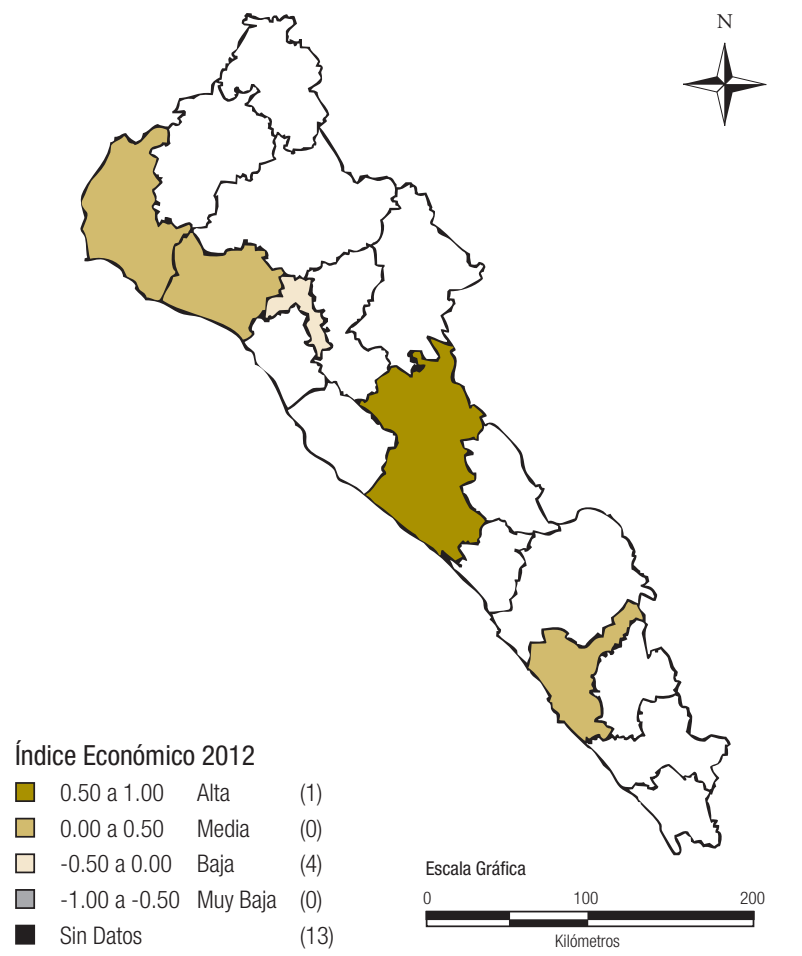

Fuente: elaboración propia

La Figura 11 nos indica el comportamiento del índice de gestión de los OOSAPyS en las ciudades del estado de Sinaloa para el año 2010; aquí está sintetizada la valoración de los veinticuatro indicadores que conforman el sistema global, distribuidos en las tres categorías: social, ambiental y económica. En esta figura se muestra la distribución del índice de gestión hídrica. Culiacán vuelve a aparecer como la ciudad hegemónica del grupo, al permanecer en el nivel alto. En cambio, Mazatlán, Los Mochis y Guasave están en nivel medio y, por último, tenemos a la localidad de Guamúchil, que ocupa el horizonte muy bajo en la medición de esta valoración de los OOSAPyS para el año 2010. Las estrategias y acciones para incrementar el desempeño de los organismos operadores en materia de la gestión del agua que se utiliza en contextos urbanos han sido
Figura 11.

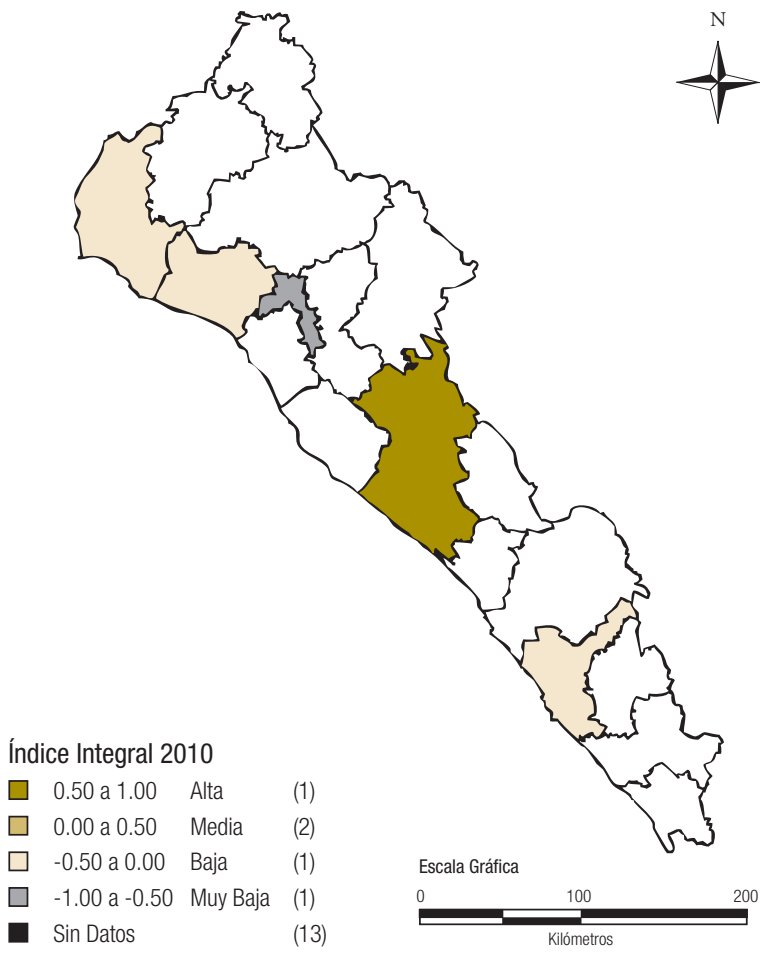

Fuente: elaboración propia

discutidas en cada apartado de las categorías social, ambiental y económica.

La valoración de la gestión de líquido vital de uso urbano para 2011 se observan en la Figura 12. La ciudad de Culiacán se ubica una vez más en el nivel alto. En el peldaño medio está la localidad de Los Mochis y en el sitio bajo se halla Mazatlán. Guasave y Guamúchil están posicionadas en el nivel muy bajo.

La valoración del índice de gestión de líquido vital de uso urbano para el 2012 se indica en la Figura 13. Culiacán se mantiene en el nivel alto. Las localidades de Los Mochis, Mazatlán, Guasave y Guamúchil no sufren cambios significativos con respecto al año anterior. Ello nos señala que han mantenido su desempeño en los indicadores de las tres categorías. 


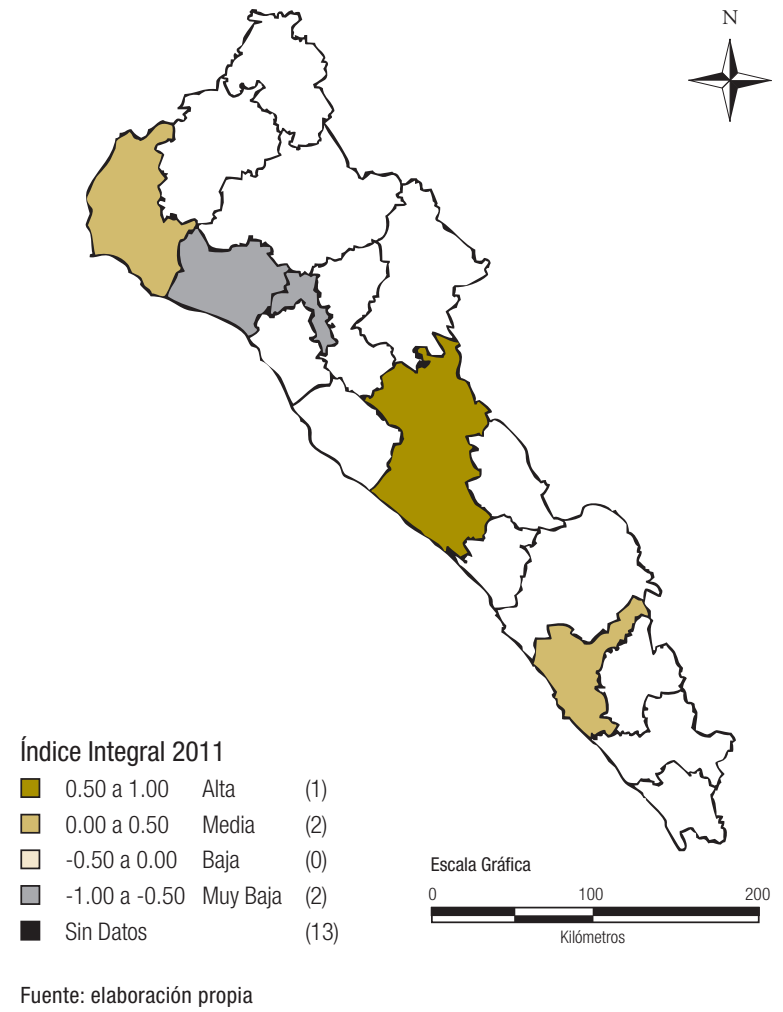

\section{Conclusiones}

La conformación de la clasificación (ordenamiento) de los OOSAPyS de las cinco ciudades del estado de Sinaloa, analizado para el período 2010-2012, nos ha obligado a la construcción del sistema básico de veinticuatro indicadores distribuidos en tres categorías: social, ambiental y económica, con ocho indicadores cada una. Ello nos llevó a comprender la dinámica del posicionamiento de cada uno de los OOSAPyS en materia de gestión de los servicios de agua potable, alcantarillado y saneamiento, al observar sus fortalezas y debilidades, con visión, principios y criterios del desarrollo sustentable.

En el estudio se observa un predominio de las ciudades que atienden una población importante

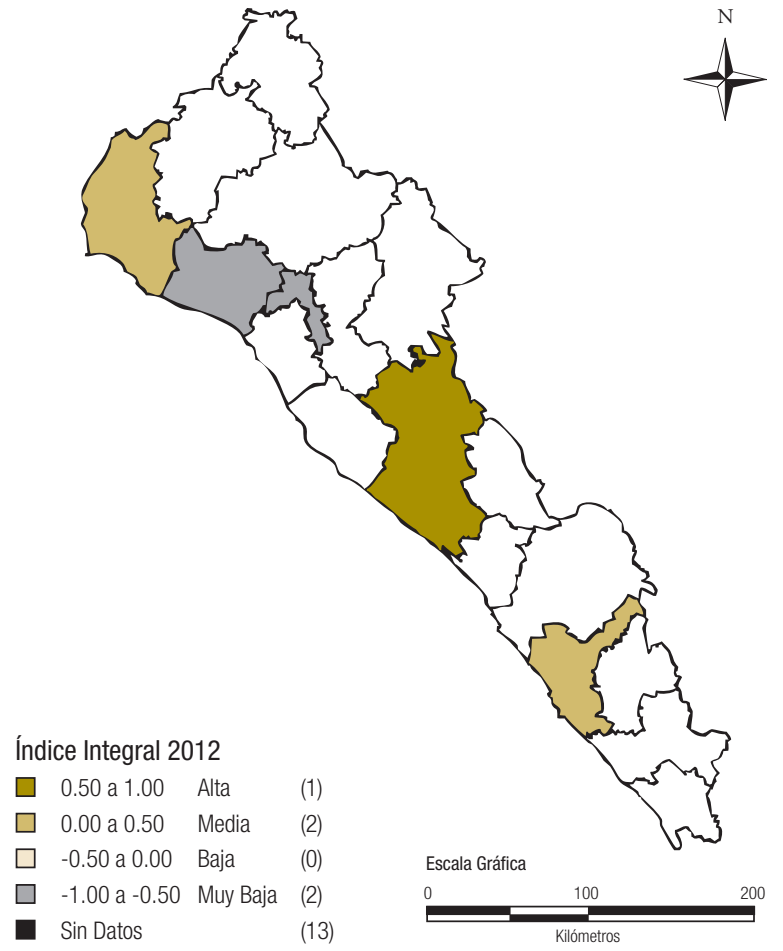

Fuente: elaboración propia

(por encima de los 400.000 habitantes) y son territorios que tienden al proceso de conurbación en su contexto urbano, como Culiacán, su capital, centro urbano que está prácticamente ligado con la ciudad de Navolato. Mazatlán es un centro de atracción turística internacional y nacional; la ciudad ya presenta un proceso conurbado con la población de Villa Unión y con la ciudad de Concordia. De igual manera, la ciudad de Los Mochis es una localidad conurbada con la ciudad y puerto de Topolobampo. La ciudad de Los Mochis también muestra el proceso de conurbación urbana con la localidad de Juan José Ríos, poblado que pertenece al municipio de Guasave.

Aquí se ve que las categorías social y ambiental aportan una proporción significativa en la valoración integral del sistema, no así la dimensión 
económica; por tanto, es necesario instrumentar acciones que mejoren los indicadores económicos: macromedición, eficiencias, tarifas y costos de producción. Tres ciudades - Culiacán, Mazatlán y Los Mochis- presentan una gran diferencia en el parámetro de población y, por lo tanto, este es un factor determinante, puesto que son los indicadores de la categoría social los que aportan la mayor proporción en la valoración integral. En cambio, en las localidades de Guasave y Guamúchil, los organismos operadores deberán instaurar acciones en la categoría económica para que mejoren sus indicadores, como macromedición, eficiencias y tarifas.

Se recomienda que las cinco localidades gestionen la posibilidad de mezclar recursos financieros del Estado, desde sus tres niveles de gobierno, para que definan estrategias y acciones orientadas hacia un manejo sustentable del agua y mejoren la calidad de los servicios que ofrecen a sus habitantes, sobre todo en materias de desinfección, potabilización y tratamiento de las aguas residuales municipales. Como una última alternativa, los OOSAPyS deben disponer de aportaciones de empresas del sector privado para que mejoren los indicadores de desempeño en las tres categorías. En otras palabras, sus acciones deben encaminarse a optimizar la cobertura de todos sus servicios y aumentar la disponibilidad de más infraestructura, todo ello para disminuir la presión que hoy día se ejerce sobre el recurso hídrico contenido en las cuencas hidrológicas, puesto que estas muestran ya una irracional sobreexplotación del acuífero donde se localizan las ciudades en estudio.

\section{Bibliografía}

Aguarón, J., y Moreno-Jiménez, J. M. (2003). The Geometric Consistency Index. Approximated Thresholds. European Journal of Operational Research, 147(1), 137-145.
Barba-Romero, S., y Pmerol, J. C. (1997). Decisiones multicriterio. Fundamentos teóricos y utilización práctica. Madrid: Servicio de Publicaciones de la Universidad de Alcalá.

Collado, J. (2008). Entorno de la provisión de los servicios públicos de agua potable en México. En R. Olivares y R. Sandoval (coords.), El agua potable en México. Historia reciente, actores, proceso y propuestas (pp. 3-28). México: Asociación Nacional de Empresas de Agua y Saneamiento de México, A. C. (ANEAS).

Comisión Nacional del Agua [Conagua]. (2010). Situación del subsector agua potable, alcantarillado y saneamiento. Recuperado de http://www.conagua.gob.mx/CONAGUA07/Publicaciones/Publicaciones/DSAPAS\%202010.pdf

Comisión Nacional del Agua [Conagua]. (2011). Situación del subsector agua potable, alcantarillado y saneamiento. Recuperado de http://www.conagua.gob.mx/CONAGUA07/Publicaciones/Publicaciones/DSAPAS\%20Edicion\%202011.pdf

Comisión Nacional del Agua [Conagua]. (2012). Situación del subsector agua potable, alcantarillado y saneamiento. Recuperado de http://www.conagua.gob.mx/CONAGUA07/Publicaciones/Publicaciones/SGAPDS-40-12.pdf

Harker, P. T. (1987). Incomplete Pairwise Comparisons in the Analytic Hierarchy Process. $\mathrm{Ma}$ thematical Modelling, 9(11), 837-848.

López, M. E., Sánchez, P., e Iglesias, C. L. (2003). Bienestar socioeconómico de los municipios gallegos: una aproximación a su medida. Revista Gallega de Economía, 12(2), 1-24.

Pena, J. A. (1978). La distancia P: un método para la medición de bienestar. Revista Española de Economía, 8(1), 49-89.

Pérez, V. E., Blancas, F. J., González, M., Guerrero, F. M., Lozano, M., Pérez, F., y Caballero, 
R. E. et al. (2009). Evaluación de la sostenibilidad del turismo rural mediante indicadores sintéticos. Investigación operacional, 30(1), 40-51.

Saaty, T. (1980). The Analytic Hierarchy process: Planning, Priority Setting, Resource Allocation. Nueva York: McGraw-Hill.

Secretaría de Medio Ambiente y Recursos Naturales [Semarnat]. (2011). Logros en el sector hídrico 2007-2010. México D. F.: Comisión Nacional del Agua.

Settle, C., Crocker, T. D., y Shogren, J. F. (2002). On the Joint Determination of Biological and Economic Systems. Ecological Economics, 42(12), 301-311.
Urzelai, A., Olazábal, M., García, G., Santa Coloma, O., Herranz, K., Abajo, B. et al. (2006). Modelización de un sistema territorial "urbano-rural" para la evaluación de su sostenibilidad. Aplicación a una zona representativa del País Vasco. Revista Internacional de sostenibilidad, tecnología y humanismo (1), 159-172.

Van den Berg, C. y Danilenko, A. (2011). The IBNET Water Supply and Sanitation Performance Blue Book. The International Benchmarking Network for Water and Sanitation Utilities Databook. Washington, D. C.: The World Bank.

Zahedi, F. (1986). The Analytic Hierarchy Process. A Aurvey of the Method and its Applications. Interfaces, 16(4), 96-108. 